Subject \& Method A hospital based, retrospective case-review study was carried out in Pediatric department, Al-Adan Hospital, State Of Kuwait. The files of all patients less than 12 year age, and for whom CT lung was performed during the last five years (20002005) were reviewed.

Results Thirty patients for whom CT lung was performed were reviewed. The main indication for requesting chest CT was persistent abnormal CX-ray findings $(90 \%)$ in a patient with uncontrolled wheeze and/or recurrent pneumonia. Findings of chest CT was the same as CX-ray findings (80\%) and more specific +/- new diagnosis $(20 \%)$. Results of chest CT influenced medical treatment only in seven of the cases (23\%) and led to surgical intervention in two patients $(0.06 \%)$, (hernia, cystic adenomatoidmalformation).

Conclusion Our data demonstrate that the result of CT lung in children doesn't alter clinical decision and management of most of the cases. Therefore CT lung should be requested mainly for the high risk patients, preferably by a pulmonologist and after discussing the chest $\mathrm{x}$-ray findings with a radiologist.

\section{ESTIMATION OF NEONATAL ENDOTRACHEAL TUBE RESISTANCE BY WATER MANOMETER}

doi:10.1136/archdischild-2012-302724.1709

'P Mallya, ${ }^{2} \mathrm{P}$ Webb, ${ }^{1,3} \mathrm{~S}$ Gupta. 'Paediatrics and Neonatal Medicine, University Hospital of North Tees, Stockton; ${ }^{2}$ Medical Physics, James Cook University Hospital, Middlesbrough: 3University of Durham, Durham, UK

Background The loss of pressures at the distal end of ET tube can be affected by the size and diameter of the ET tube and the flow rates.

Aim To study in vitro the pressure drop using varying sizes of ET tubes commonly used in preterm infants at different lengths, flow rates using different gases.

Methods We used Portex tubes for this in-vitro study. A water manometer (scale $0-300 \mathrm{~mm}$ ) was used to measure the pressure drop across ET tube. We used two different lengths (7 and $14 \mathrm{cms})$; different sizes $(2.5 ; 3.0 ; 3.5$ and $4.0 \mathrm{~mm}$ ), different flow rates (4 to 11 litres/min) and two different gases (medical air and 100\% oxygen) to assess the resistance across the ET tube. SPSS version 17 $®$ was used for statistical analysis. Data presented as mean(SD).

Results

1. There was no difference in the pressure drop at lengths of $7 \mathrm{cms}$ and $14 \mathrm{cms}$ respectively using air [120.6 (66.12) vs.127.3 (68.74) $\mathrm{cms}_{2} \mathrm{O}$; $\left.\mathrm{p}=0.297\right]$; but with $100 \%$ Oxygen there was statistically significant increase at $14 \mathrm{~cm}$ compared to $7 \mathrm{~cm}$ length [146.73 (72.94) vs. $130.48\left(72.94 \mathrm{cms} \mathrm{H}_{2} \mathrm{O}\right.$; $\mathrm{p}=0.015]$ at similar flow rates.

2. At all flow rates there was no difference in pressure drop at different tube diameters using air or $100 \%$ oxygen

3. There was a statistically significant $(p<0.05)$ increase in pressure drop at flow rates increasing from 4 to 11 litres $/ \mathrm{min}$.

Abstract 1709 Table 1 Comparison of pressure drop with air and oxygen

\begin{tabular}{lll}
\hline Flow (I/min) & $\begin{array}{l}\text { Pressure drop with Air } \\
\mathbf{C m s H}_{\mathbf{2}} \mathbf{0}(\mathbf{M e a n} / \mathbf{S D})\end{array}$ & $\begin{array}{l}\text { Pressure drop with oxygen } \\
\mathbf{C m s H}_{\mathbf{2}} \mathbf{0} \text { (Mean/SD) }\end{array}$ \\
\hline 4 & $42.4 / 6.4$ & $55 / 26.3$ \\
5 & $58.1 / 8.3$ & $74.5 / 20.3$ \\
6 & $77.9 / 10.8$ & $97.7 / 16.0$ \\
7 & $101.6 / 13.5$ & $129 / 14.6$ \\
8 & $125.9 / 16$ & $162.1 / 18.9$ \\
9 & $156.7 / 20$ & $198 / 25.6$ \\
10 & $197.9 / 25.7$ & $236.2 / 33$ \\
11 & $227.7 / 59.3$ & $182.4 / 129$ \\
\hline
\end{tabular}

Conclusion The results from this study could be utilised to optimise ventilation settings to achieve the desired pressure delivery using air or oxygen at different flow rates and with varying ETtube sizes.

\section{SEROPREVALENCE OF CHLAMYDIA PNEUMONIAE AND MYCOPLASMA PNEUMONIAE IN CHILDREN WITH CHRONIC COUGH}

doi:10.1136/archdischild-2012-302724.1710

${ }^{1}$ S Asilsoy, ${ }^{2}$ E Bayram, ${ }^{3} \mathrm{D}$ Can. ${ }^{1}$ Pediatric Allergy, Başkent Universitesi, Adana; ${ }^{2} D o k u z$ Eylül University; ${ }^{3}$ Dr Behcet Uz Çocuk Hastanesi, Izmir, Turkey

Background Chronic cough is one of the most common symptoms in children. Postinfectious etiologies plays an important role in chronic cough in childhood. The pathogenesis of the postinfectious cough may be related persistant inflammation and the epithelial damage in the upper and lower airways, with or without transient airway hyperresponsiveness. We evaluated Mycoplasma pneumoniae and Chlamydia pneumoniae serology and treatment in children referred with chronic cough.

Methods This study enrolled 41 children between 6 and 14 years of age having cough which lasted than 4 weeks. They were evaluated according to American College of Chest Physicians guideline. Pulmonary function test and chest x-ray were performed to all patients. M. pneumoniae and C. pneumoniae serologies were analayzed by ELISA. They were reevaluated with 2 to 4 weeks intervals until cough disappeared.

Results The study included 41 children, 27 of whom were female (65.9\%). The mean age was $8.00 \pm 1.96$ year. M. pneumoniae IgM positivity was found in $17.07 \%(7 / 41)$ of patients, C. pneumoniae IgM positivity in $2.85 \%$ (1/35), M. pneumoniae IgM and/or IgG positivity in $41.46 \%(17 / 41)$, C. pneumoniae IgM and/or IgG positivity in $25.7 \%$ (9/35). Symptoms were not improved alone with clarithromycine treatment so inhaled/nasal steroids were added according to diagnosis.

Conclusion In children with chronic chough, aged 6 to 14 years old, M. pneumoniae and C. pneumoniae play important roles in the etiology. Clarithromycine alone may not be enough in the treatment of chronic cough due to these agents, so the treatment should be planned according to clinical findings.

\section{THE RELATIONSHIP BETWEEN THE FIRST EPISODE OF WHEEZING AND MATRIX METALLOPROTEINASES-9 AND -2 AND TIMP-1 LEVELS IN PRETERM INFANTS}

doi:10.1136/archdischild-2012-302724.1711

${ }^{1} \mathrm{RG}$ Sezer, ${ }^{1} \mathrm{~A}$ Bozaykut, ${ }^{2} \mathrm{~A}$ Tanju, ${ }^{2} \mathrm{G}$ Aydemir, ${ }^{1} \mathrm{LP}$ Seren, ${ }^{3} \mathrm{~S}$ Hira, ${ }^{3} \mathrm{O}$ Ozcan. ${ }^{1}$ Department of Pediatrics, Zeynep Kamil Maternity and Childrens' Disease Training and Research State Hospital; '2Department of Pediatrics; ${ }^{3}$ Department of Biochemistry, GATA Teaching Hospital, Istanbul, Turkey

Background Elevated concentrations of matrix metalloproteinases (MMP) have been associated with neonatal morbidity. There are no data on the serum levels of MMP-2, MMP-9, tissue inhibitors of matrix metalloproteinase (TIMP-1) from preterm infants recovering from these morbidities. We aimed to compare MMP-2, MMP-9, and TIMP-1 levels in preterm and term infants hospitalized with their first episode of wheezing.

Methods We prospectively evaluated 18 preterm infants with a history of chronic lung disease, respiratory distress syndrome and 14 age- and sex- matched term infants who were admitted for a first episode of wheezing. We quantified total serum concentrations of MMP-2, MMP-9, and TIMP-1 to assess whether these serum markers levels were associated with wheezing with a history of oxygen therapy during the neonatal period.

Results Upon hospitalization for the first episode of wheezing, MMP-2 and TIMP-1 levels were higher in preterm infants than in term infants. 

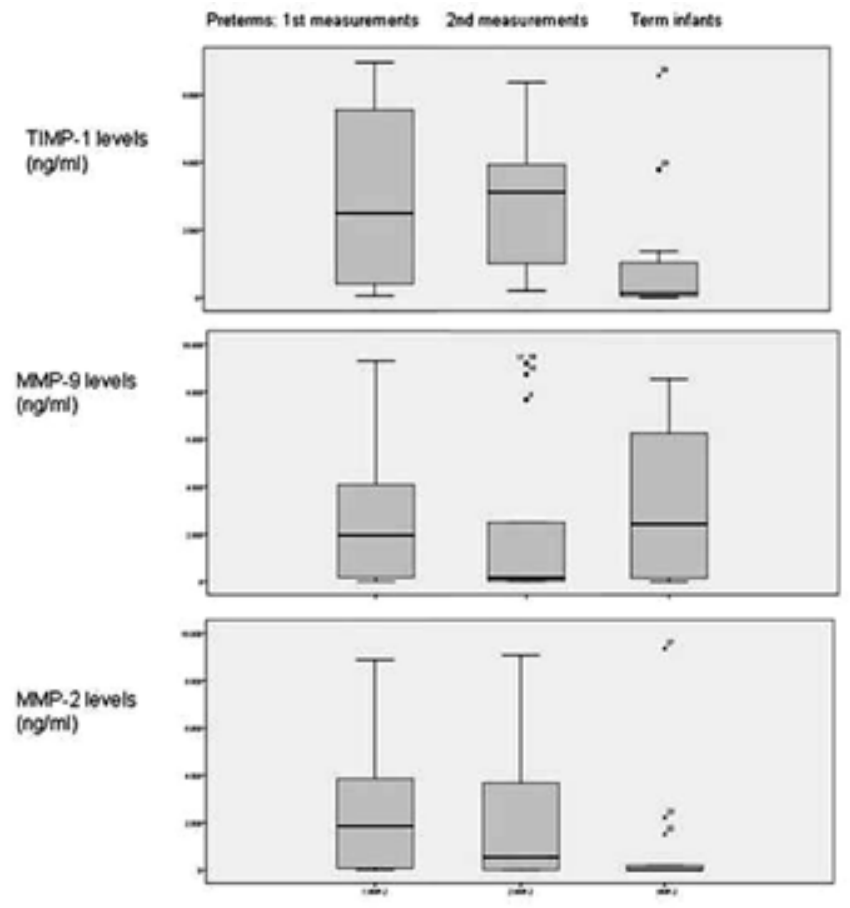

Abstract 1711 Figure 1 Comparison of MMP-2, MMP-9, and TIMP-1 levels

In contrast, there was no significant relationship between MMP-9 levels or the MMP-9/TIMP-1 ratio between preterm and term infants (univariate analysis). The area under the ROC curve for MMP-2 was 0.70 (95\% CI 0.51-0.89, p=0.04). The area under the curve for TIMP-1 was 0.78 (95\% CI 0.61-0.94, p=0.007). MMP-9, MMP-2, and TIMP-1 levels did not correlate with gestational age, gender, severity of wheezing.

Conclusion Elevated serum MMP-2 and TIMP-1 levels appear to increase the risk for wheezing. Further studies will be required to determine whether therapeutic inhibitors will prevent recurrent lung morbidities in preterm infants.

\section{SERUM ELECTROLYTES VARIATIONS IN TREATED PATIENTS WITH MODERATE ASTHMA EXACERBATION}

doi:10.1136/archdischild-2012-302724.1712

ML Neamtu, AC Brumar. Pediatric Clinic Hospital, Lucian Blaga University of Sibiu, Sibiu, Romania

Background Salbutamol induces stimulation of beta ${ }_{2}$-receptors resulting in hypokalemia. Corticosteroids also induce plasma electrolytes variations.

Aims

1. To identify blood electrolytes changes following low dose inhaled short-acting beta ${ }_{2}$-agonists;

2. To evaluate if concomitant inhaled corticosteroids treatment can amplify serum electrolytes changes.

Methods We analyzed all children admitted for moderate asthma exacerbation during 6 months period. Inclusion criteria: children between $5-18$ years of age; PEF $>50-75 \%$ of predicted value; serum electrolytes normal ranges. Exclusion criteria: previously treated patients with Salbutamol; Salbutamol hypersensitivity; asthma exacerbation severity levels. The patients were divided into 2 groups: $1^{\text {st }}$ group comprised those treated with beta ${ }_{2}$-agonists and $2^{\text {nd }}$ group is represented by paediatric patients concomitantly treated with beta $_{2}$-agonists and corticosteroids. Both groups were homogenous regarding age and sex ratio. During hospitalization, patients received standard low dose of Salbutamol by metered dose inhaler (MDI) and inhaled Fluticasonum propionate using spacer device with mouthpiece. Included patients were assessed for electrolytes serum levels before treatment and 72 hours after therapy. Data was analyzed statistically using independent sample $\mathrm{T}$ test, skewness, kurtosis.

Results Among 269 admitted patients, 175 children fulfilled inclusion criteria. Both groups structure: 92 children in $1^{\text {st }}$ group, 83 in $2^{\text {nd }}$ group. Authors found for both groups a significant decreasing of serum kalium after beta $_{2}$-agonists treatment ( $p$ value $\left.=0,010\right)$. The study didn't confirm a significant variation of serum electrolytes in $2^{\text {nd }}$ group as compare to $1^{\text {st }}$ group.

Conclusions Study confirmed significant hypokalemia after 72 hours inhaled treatment with beta $_{2}$-agonists; corticosteroids didn't modify kalium level in association with beta ${ }_{2}$-agonists.

\section{REDUCING ASTHMA CLINIC ATTENDANCE USING POSTAL SURVEY WITH MOBILE TEXTING FEEDBACK}

doi:10.1136/archdischild-2012-302724.1713

'LM Perrem, ${ }^{2 P}$ Manning, 'MB 0'Neill. 'Mayo General Hospital, Castlebar; '2Mullingar Regional Hospital, Mullingar, Ireland

Background and Aim Attempting to reduce unnecessary attendances of well patients at outpatient clinics is prudent. This study evaluated the Asthma Control Test (ACT)t and Respiratory Proforma, with feedback through mobile texts, in children with Asthma, to determine attendance at clinic or not.

Methods Patients between 4 and 11 years with a diagnosis of asthma were eligible for inclusion. The parent was surveyed, by ppost, 2 weeks prior to the clinic date and asked to complete the Asthma Control Test (ACT) and a Respiratory Proforma which assessed UACS symptoms, medication usage inclusive of intensification episodes and medical concerns. Mobile telephone numbers were requested. Parents mailed their responses in a supplied stamped envelope supplied. Respondents were divided into 2 categories a) ACT score greater than 19 and a non concerning Respiratory Proforma, who were texted not to attend the clinic but supplied with another outpatient appointment and b) the remainder were texted to attend the clinic.

Results Over 6 clinics the parents of 77 eligible children were surveyed. Fifty eight (75\%) replied of whom 38 (66\%) were well and did not attend the clinic but rebooked. Of 20 who attended, 6 had new symptoms of UACS and 3 had pneumonia. Of 19 who did not reply 7 came to clinic with completed questionnaires, 5 had good control. Ten did not attend the clinic or complete the questionnaire.

Conclusion Asthma care through postal survey with mobile text feedback is an option in the outpatient setting.

\section{ABILITY OF SELF-INFLATING BAGS (SIB) TO DELIVER SUSTAINED INFLATIONS}

doi:10.1136/archdischild-2012-302724.1714

'M Thio, 1,2JA Dawson, ${ }^{3} \mathrm{TJ}$ Moss, ${ }^{3} \mathrm{SB}$ Hooper, ${ }^{1,2} \mathrm{PG}$ Davis. 'Newborn Services, The Royal Women's Hospital; ${ }^{2}$ Murdoch Children's Research Institute; ${ }^{3}$ The Ritchie Centre, MIMR, Monash University, Melbourne, VIC, Australia

Background and Aims In neonatal resuscitation, the use of a sustained inflation (SI) after birth may facilitate lung recruitment. We aimed to assess the ability of several SIB to deliver a SI.

Method In a newborn preterm lamb, we compared 4 different SIB devices fitted with a PEEP valve against a T-piece, using a flow of $8 \mathrm{Lpm}$. Four operators aimed to give 3 targeted SI of $20 \mathrm{cmH}_{2} \mathrm{O}$ (displayed on a manometer) for 30 seconds. The study was repeated with the PEEP valve removed and again with no flow. 